\title{
Effect of non-surgical periodontal therapy on superoxide dismutase levels in gingival tissues of chronic periodontitis patients: A clinical and spectophotometric analysis
}

\author{
Laxmi Sukhtankar, Anita Kulloli, Rahul Kathariya* and Sharad Shetty \\ Department of Periodontics and Oral Implantology, Dr. DY Patil Dental College and Hospital, Pimpri, Pune, \\ Maharashtra, India
}

\begin{abstract}
.
BACKGROUND: Superoxide dismutase (SOD), an antioxidant acting against superoxide (oxygen radical, $\mathrm{O}_{2}^{-}$), it is released in inflammatory pathways and causes connective tissue breakdown. Increased SOD activity in inflamed gingiva may indicate increased $\mathrm{O}_{2}^{--}$radical generation by neutrophils and other inflammatory cells at the diseased site. The aim of the study was to evaluate the effects of non-surgical periodontal therapy (NSPT) on SOD levels in gingival tissues of chronic periodontitis patients.

METHODS: Forty subjects: 20 periodontally healthy (Control) and 20 chronic periodontitis (Test); age range 24-55 years were recruited. Gingival tissue samples were collected by excising the inner lining of the periodontal pocket at baseline (prior to non-surgical periodontal therapy) and 2 months post therapy. In controls, tissue samples were obtained immediately after tooth extraction scheduled for orthodontic reasons. Clinical parameters included probing depth, clinical attachment level, gingival index, bleeding index, plaque index. SOD activities were assessed spectrophotometrically at baseline and 2 months post NSPT, results were analysed statistically.

RESULTS: At baseline, patients with chronic periodontitis had higher mean SOD activity $(2.73 \pm 1.36)$ than the control subjects $(1.12 \pm 1.13)$ with $p=0.00003(p<0.05)$. At 2 months post NSPT median SOD level $(1.00)$ had come close to median SOD value of control group (0.85); $p=0.99(p>0.05)$. The resolution of inflammation with successful NSPT resulted in decreased SOD levels as in control group. Clinical parameters in patients with chronic periodontitis showed a significant improvement 2 months post NSPT $(p<0.05)$.

CONCLUSION: Non-surgical periodontal therapy significantly improves the clinical parameters and restores previously increased SOD levels to normal in chronic periodontitis patients.
\end{abstract}

Keywords: Non-surgical periodontal therapy, superoxide dismutase, chronic periodontitis, gingiva, neutrophils

\section{Introduction}

Chronic periodontitis (CP) is initiated by sub-gingival biofilm. However, the progression of destructive

*Corresponding author: Rahul Kathariya, Department of Periodontics and Oral Implantology, Dr. DY Patil Dental College and Hospital, Pimpri, Pune - 400018, Maharashtra, India. Tel.: +91 8983370741; E-mail: rkathariya@gmail.com. disease appears to be dependent upon abnormal host response to the biofilm organisms [1]. The periodontitis phenotype is characterized by hyper inflammation involving excess oxygen free radical release by inflammatory cells mostly polymorphonuclear leucocytes (PMNs) [1]. In recent years, a collective term 'Reactive Oxygen Species' (ROS) or 'reactive oxygen intermediates' is been adopted [1]. ROS arise primarily from 'respiratory burst' characterized by ac- 
tivated phagocytes during the host defence mechanism. While most ROS have extremely short half-lives $\left(10^{-9}-10^{-10}\right.$ seconds), they cause substantial damage to the host cells by initiating free radical chain reactions [1].

Protection against such species is provided by antioxidants (AOs). Many of which, are released locally at sites of inflammation by PMNs and other cells [2]. Antioxidants are those substances which when present at low concentrations, compared to oxidisable substrate, will significantly delay or inhibit oxidation of that substrate [3]. Superoxide Dismutase (SOD) is one of an important $\mathrm{AO}$ protecting the cell against the deleterious effects of ROS.

Body's extracellular fluids contain extremely low levels of SOD activity [2]. Thus, saliva would be its poor source [2]. The human periodontal ligament has shown to possess increased SOD activity [2]. This might afford biological protection against ROS, particularly $\mathrm{O}_{2}^{-}$, during the inflammatory response [2]. Increased SOD activity in inflamed gingiva from chronic periodontitis patients may indicate the increased $\mathrm{O}_{2}^{-}$ generation by PMNs invaded at the diseased sites [2]. Moreover, a significant reduction of GCF SOD activity was found in gingival tissue adjacent to deep periodontal pockets following non surgical periodontal therapy [4].

Wei et al. [5] reported, non-surgical periodontal therapy controlled and restored the subject's lipid peroxidation levels, total oxidant status and superoxide dismutase levels in serum, saliva and gingival crevicular fluid. However, there are no reports of these AO in the gingival tissue. Hence, in this study, we evaluated SOD levels in gingival tissues and not in GCF/Saliva. To our knowledge, no study has reported the effect of periodontal therapy on gingival SOD levels in chronic periodontitis. Thus, the purpose of the present study was to investigate the effect of non-surgical periodontal therapy on gingival SOD levels in chronic periodontitis patients.

\section{Materials and methods}

This study was carried out from August 2011 to December 2011 . The study population consisted of 40 age and gender balanced subjects (20 males, 20 females, age range: $24-55$ years) attending the Out Patient section, Department of Periodontics and Oral Implantology, Dr. DY Patil Dental College and Hospital, Pimpri, Pune. Written informed consent was obtained from those who agreed to participate voluntarily. Ethical clearances were obtained from the institution's ethical committee and review boards. Subjects having minimum 20 natural teeth were randomly selected and categorized into 2 groups: Control group- 20 periodontally healthy and Test group- $20 \mathrm{CP}$ subjects based on the following criteria.

Control group: Subjects with no history of periodontal disease, with probing depths of $\leqslant 3 \mathrm{~mm}$ and clinical attachment level (CAL) of $\leqslant 1 \mathrm{~mm}$ with full mouth bleeding scores less than $10 \%$. Test Group: Subjects having $\geqslant 5 \mathrm{~mm}$ probing pocket depth (PD) or attachment level of $\geqslant 2 \mathrm{~mm}$ in at least four teeth, with radiographic evidence of bone loss.

Patients with any systemic disease, history of antibiotic and anti-inflammatory therapy any periodontal procedures (including scaling) in the previous 6 months, aggressive periodontitis, infectious conditions other than periodontitis, history of smoking or use of tobacco in any form, or patients consuming antioxidant vitamins or patients with poor oral hygiene maintenance were excluded from the study.

\section{Clinical parameters}

Clinical parameters included: Plaque Index (PI) (Silness and Loe 1964), Gingival Index (GI) (Loe and Silness 1963), Gingival bleeding Index (GBI) (Ainamo and Bay 1975), PD and Clinical attachment level (CAL). The clinical parameters in test group were measured at baseline (prior to NSPT) and at 2 months post therapy. Clinical parameters were recorded using UNC 15 periodontal probe and radiographs (IOPA/OPG) were taken as diagnostic aid.

\section{Methodology}

\subsection{Non-surgical periodontal therapy}

Deep subgingival ultrasonic instrumentation using a magneto-restrictive ultrasonic device was done for each patient (test group) at baseline and at 2 months. Scaling and root planing was completed in an unlimited time appointment. Post scaling and root planning subjects were instructed to brush their teeth twice a day and rinse after every meal. Chlorexidine mouthwash was prescribed for these patients to use for two weeks. At two months follow up, a full periodontal reassessment was carried out after which, plaque control and oral hygiene instructions were repeated. 


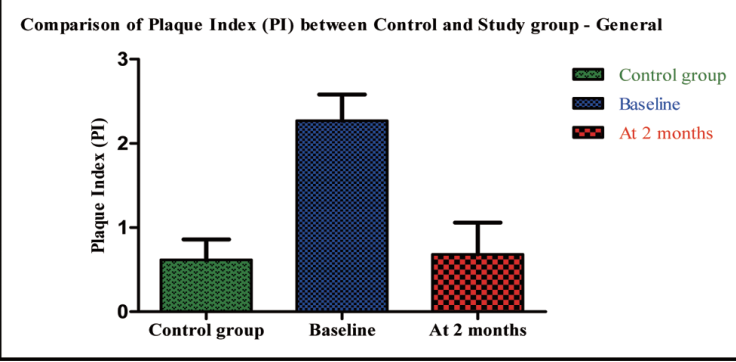

Fig. 1. Comparison of plaque index (PI) between control and study groups at baseline and at 2 months. (Colours are visible in the online version of the article; http://dx.doi.org/10.3233/DMA-130978)

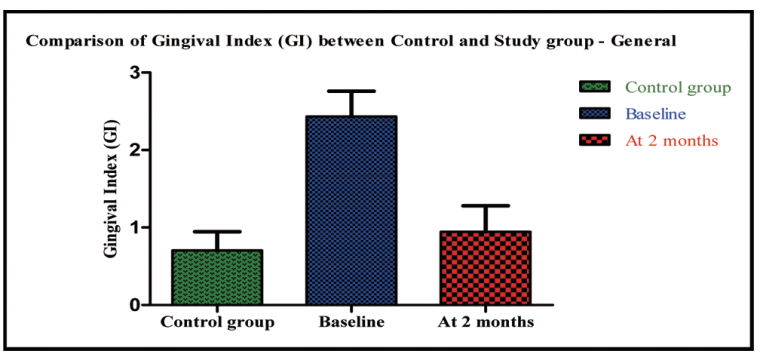

Fig. 2. Comparison of gingival index (GI) between groups at baseline and at 2 months. (Colours are visible in the online version of the article; http://dx.doi.org/10.3233/DMA-130978)

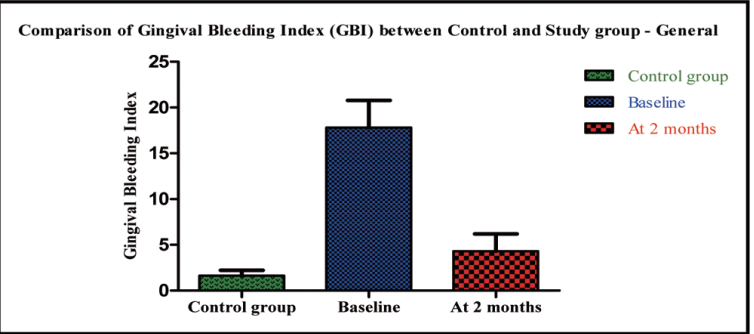

Fig. 3. Comparison of gingival bleeding index (GBI) between groups at baseline and at 2 months. (Colours are visible in the online version of the article; http://dx.doi.org/10.3233/DMA-130978)

\subsection{Sampling of gingiva}

Control group: teeth to be extracted for orthodontic treatment were selected for the study. Immediately after extraction, the gingiva surrounding the socket was excised in small pieces. Test group: Gingival tissue samples were collected at baseline prior to NSPT and at 2 month. The samples were collected by excising the inner lining of the periodontal pocket along with some amount of connective tissue. All the gingival tissue samples (20-50 mg), were washed out from contaminated blood with saline solution at $+4^{\circ} \mathrm{C}$ immediately after excision and dried with filter papers. The tissue samples were preserved in $20 \mathrm{mM}$ tris HCL buffer placed in $1.5 \mathrm{ml}$ Eppendorf tubes. Tissue samples were sent to laboratory (Department of Microbiology and Immunology, MMNH Institute of Dental Sciences and Research Centre, Belgaum) in gel coolant packs $\left(-20^{\circ} \mathrm{C}\right)$. All the samples were stored at $-20^{\circ} \mathrm{C}$ until analysis [2]. SOD activity was assayed based on reduction of nitroblue tetrazolium (NBT) by $\mathrm{O}_{2}^{--}$anion produced by Xanthine oxidase. The formazan that was formed subsequent to electron transfer to NBT by Xanthine oxidase was spectrophotometrically determined at $560 \mathrm{~nm}$. One unit (U) of SOD was defined as the amount of protein that inhibits $50 \%$ of the rate of NBT reduction.

\subsection{Procedure}

One millilitre of $20 \mathrm{mM}$ tris-HCl buffer ( $\mathrm{pH}$ 6.5) was added after the samples were homogenized in equal amounts of cold distilled water for $3 \mathrm{~min}$. The homogenates were centrifuged at $1500 \mathrm{rpm}$ for $30 \mathrm{~min}$ to remove debris. Clear upper supernatant fluids were collected and assayed. All the procedures mentioned above were performed at $+4^{\circ} \mathrm{C}$.

\section{Statistical analysis}

Descriptive statistics were expressed as mean \pm standard deviation (SD) for each parameter at different time interval. The difference between each pair of measurement was then calculated. Data were analyzed using Student's ' $t$ ' test for paired and unpaired observations to assess changes obtained within and between groups. Mann Whitney U-test, a non-parametric test was used when there was more variation within the groups. Wilcoxon matched pair signed Rank test, a non-parametric test was used when there was more variation within the groups and when observations were paired. Chi-square test: a non-parametric test was used to check the distribution among males and females in the groups.

\section{Results}

\subsection{Clinical parameters}

Mean \pm standard error values of clinical parameters are given in Table 1. All of the clinical parameters were significantly higher in the test group compared to the 
Table 1

Effect of NSPT on clinical parameters and SOD levels within study group

\begin{tabular}{lcccc}
\hline Study group & Baseline & 2 months & $t$-statistics \\
\hline PI (Mean \pm SD) & $2.27 \pm 0.31$ & $0.68 \pm 0.37$ & 19.53 & $0.00^{*}$ \\
GI (Mean \pm SD) & $2.43 \pm 0.32$ & $0.94 \pm 0.33$ & 18.51 & $0.00^{*}$ \\
GBI (Mean \pm SD) & $17.81 \pm 2.98$ & $4.30 \pm 1.89$ & 34.20 & $0.00^{*}$ \\
PD (Mean \pm SD) & $6.7 \pm 0.97$ & $5.7 \pm 0.97$ & 5.21 & $0.00^{*}$ \\
CAL (Mean \pm SD) & $6.65 \pm 0.87$ & $5.7 \pm 0.97$ & 4.25 & $0.0004^{*}$ \\
SOD (Mean \pm SD) & $2.78 \pm 1.44$ & $0.94 \pm 0.57$ & 238.0 (Sum of signed ranks) & $0.0001^{*}$ \\
\hline
\end{tabular}

* Statistically significant.

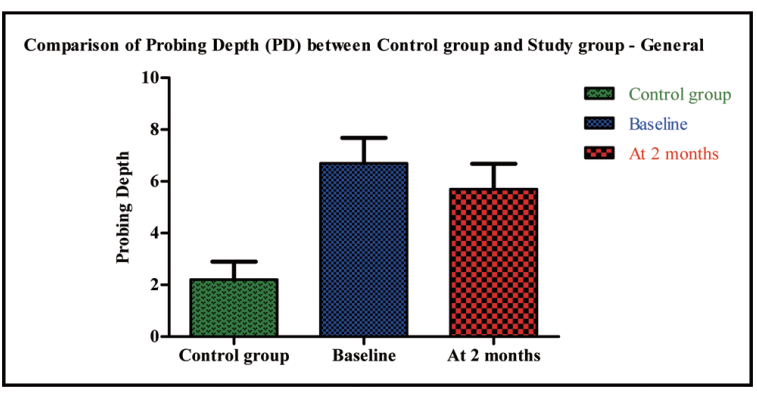

Fig. 4. Comparison of probing depth (PD) between groups at baseline and at 2 months. (Colours are visible in the online version of the article; http://dx.doi.org/10.3233/DMA-130978)

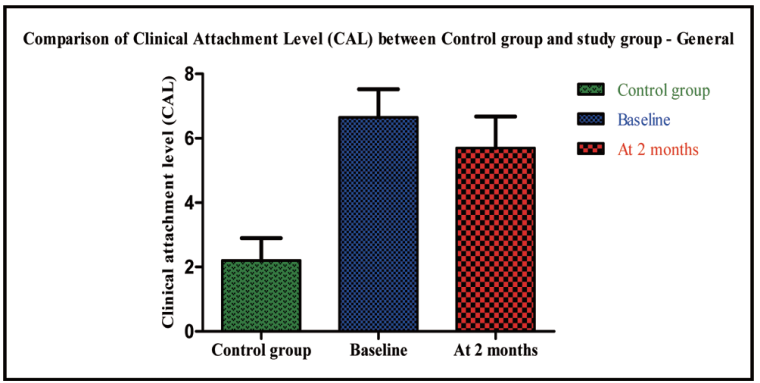

Fig. 5. Comparison of clinical attachment level (CAL) between groups at baseline and at 2 months. (Colours are visible in the online version of the article; http://dx.doi.org/10.3233/DMA-130978)

control group $(p<0.05)$ (Figs $1-5$ and Table 1$)$. The success of NSPT was confirmed by the observed reductions in plaque scores (Fig. 1 and Table 1), gingival scores (Fig. 2 and Table 1), percentage of sites positive for bleeding scores $(p<0.0001)$ (Fig. 3 and Table 1), PPD $(p<0.0001)$ (Fig. 4 and Table 1) and CAL (Fig. 5 and Table 1)

\subsection{Laboratory findings}

Mean baseline gingival SOD scores of control and test groups were statistically highly significant with $p=0.00003^{* *}(p<0.05)$ (Fig. 6). At 2 months post NSPT the mean gingival SOD score of control and test groups showed no statistical significant differ-

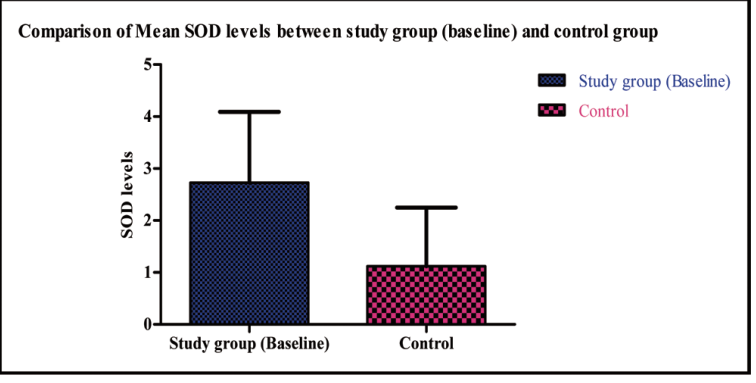

Fig. 6. Comparison of mean SOD levels between study and control groups at baseline. (Colours are visible in the online version of the article; http://dx.doi.org/10.3233/DMA-130978)

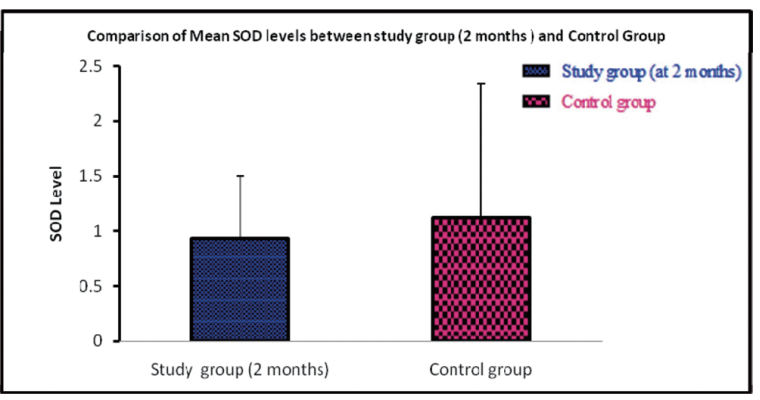

Fig. 7. Comparison of mean SOD levels between study and control groups at 2-month post NSPT. (Colours are visible in the online version of the article; http://dx.doi.org/10.3233/DMA-130978)

ence between them with $p=0.99$ ( $p>0.05$ ) (Fig. 7 and Table 1). However only the median gingival SOD scores between study group at baseline and at 2 months showed statistically significant difference with $p=$ 0.0001 ( $p$ value < 0.05) (Fig. 8).

\section{Discussion}

Within mammalian tissues the most significant $\mathrm{AO}$ is perhaps SOD which protects the tissue against the deleterious effects of ROS, ensuring that $\mathrm{O}_{2}^{--}$is efficiently converted to $\mathrm{H}_{2} \mathrm{O}_{2}$ and $\mathrm{O}_{2}$ [2].

To the best of our knowledge, this is the first study to investigate the effects of non-surgical periodontal ther- 


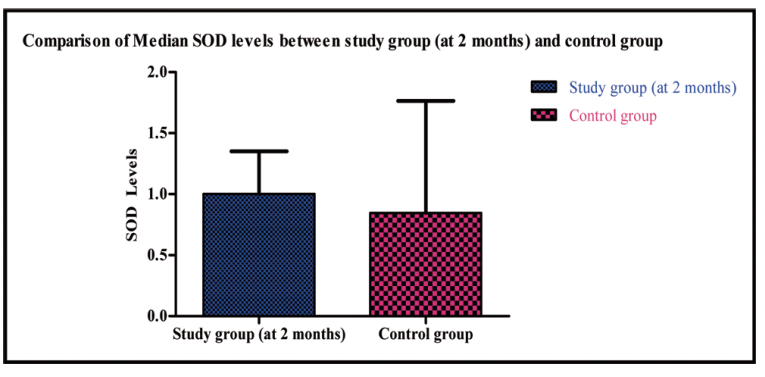

Fig. 8. Comparison of median SOD levels between control and study groups at 2 month post NSPT. (Colours are visible in the online version of the article; http://dx.doi.org/10.3233/DMA-130978)

apy on gingival SOD levels. The present study evaluated the effect of NSPT on clinical parameters and gingival SOD activity in chronic periodontitis patients compared with systemically healthy individuals. The resolution of periodontal inflammation with NSPT resulted in decreased gingival SOD levels, correlated with those found in the control group [6].

SOD is released in the body as homeostatic mechanism to protect the tissues. It is a metalloenzyme whose active centre is occupied by copper and zinc and sometimes manganese or iron. SOD is a powerful antioxidant, produced endogenously as an intracellular enzyme. It is liberated essentially in every cell of the body [7]. Pryor et al. in 1986 suggested that, the availability of SOD has provided a tool that allows testing physiological processes for the involvement of superoxide ion [8]. Increased SOD level in inflamed gingiva from chronic periodontitis patients may indicate the increased superoxide ion generation by PMNs invaded at the disease site. This increase in superoxide ion may lead to increase oxidative stress, which in turn caused an increased need for SOD production to establish the ROS-AO balance to protect the tissue. Joanny, Menvielle-Bourg in 2005 observed that powerful natural antioxidant enzyme (SOD) acts at the very source of the chain reaction resulting in reactive types of oxygen. Therefore, it constitutes the first and one of the main links of the defence process defending the cells against the hazardous effects of ROS [9]. Jacoby et al. in 1991, did the biochemical analysis of periodontal soft tissues cleaved from freshly extracted human teeth, the results of the study showed that the human periodontal ligament contained about twice as much SOD activity as human skin [10]. In another study it was showed that gingival SOD levels increased in inflamed gingiva of chronic periodontitis patients indicating the increased $\mathrm{O}_{2}^{-}$generation by PMNs invaded at the disease site [11] $\mathrm{Na}$ et al. [12] concluded that
Mn-SOD was markedly increased in the inflammatory gingival tissue proportional to the severity of inflammation, the authors also stated $\mathrm{Cu} / \mathrm{Zn}$ SOD was also increased but not as much as Mn-SOD.

To the best of our knowledge, no reports are available in literature regarding the effects of periodontal therapy on the SOD levels in chronic periodontitis patients. It is known that SODs are mainly found in all cells and tissues and there is little SOD activity in the extracellular fluids [13]. Bauer and Bauer [14] localized SOD within human periodontal ligament, and concluded that it represent an important defence mechanism within gingival fibroblasts against superoxide release. However, Akalin et al. [2] reported as SOD activity in GCF was negligent, signifying that SOD defence may be weak in GCF. Therefore, we selected gingival tissue samples for the analysis of SOD activity in our study. Systemically healthy patients were included to eliminate any confounding factor which could modify the results of the study. Igari et al. [15] found fourfold higher SOD concentration in synovial fluid from rheumatoid arthritis patients. Kimura et al. [16] suggested strong relationship between the serum concentration of extracellular (EC) SOD and the severity of both microvascular and macrovascular diabetic complications, and that serum EC-SOD concentration levels may be a marker of vascular injury.

In the present study, we excluded smokers, because according to Agnihotri et al. [17] cigarette smoking is associated with significant decrease in SOD levels. Garg et al. [18] observed the delicate balance between ROS and tissue concentrations of antioxidants may be disturbed by various factors including smoking. Elevated levels of ROS stimulate neutrophils to upregulate the adhesion integrins, leading to their increased accumulation in tissues and a local sealing off of antioxidant enzymes, such as SOD, catalyse and protease inhibitors, as observed by Ryder et al. [19]. Consequent to this there is degradation and collagenolysis of ground substance or increased stimulation of excessive proinflammatory cytokines through nuclear transcription factor kappa B activation or an increased production of prostaglandin $\mathrm{E}_{2}$ via lipid peroxidation and superoxide release; all are linked to bone resorption and can aggravate periodontitis [20]. Individuals with history of prolonged use of medications (antibiotic, anti-inflammatory therapy and antioxidant vitamins) were excluded from the study to eliminate the influence of the same on the course of periodontal disease activity, which could intern influence the SOD activity in the gingival tissues [2]. About $75 \%$ of patients 
with localized aggressive periodontitis (LAP) exhibit a PMN chemotactic defect that can impair the ability to phagocytose and kill the microorganism in the peripheral blood [21]. Like LAP patients, generalized aggressive periodontitis (GAP) have a neutrophil chemotactic defect and reduced phagocytosis [2]. Asman et al. showed that various PMN dysfunction play a key role in the progression of early onset forms of periodontitis, with impairment in leukocyte chemotaxis and adherence mechanism, and showed the increased release of free oxygen radicals from activated PMN cells in juvenile periodontitis [22]. Hence, subjects with a known history of aggressive periodontitis or potential risk of being diagnosed with it were excluded from the study.

The reduction in the clinical parameters in the present study suggests the importance of NSPT in its reduction. At baseline, the mean gingival SOD scores in control and test groups were $1.12 \pm 1.13$ and $2.73 \pm$ 1.36 respectively, with the difference of $1.61 \pm 0.23$. It has been shown that SOD activity increased directly after occurrence of oxidative stress as shown by Godin and Wohaieb [23]. Our results are in accordance with the Godin et al. Akalin et al. [2] showed SOD activity increase in gingival tissue in chronic periodontitis patients. This increase may be due to the occurrence of increased oxidative stress and the increased need for oxidant/AO balance and protection. Our results were in accordance with that of Akalin's study. Comparison of median gingival SOD activity level scores within study group (baseline and at 2 months) showed statistically significant difference. As the resolution of inflammation with successful NSPT resulted in a decrease in SOD activity reaching the levels found in control subjects. This suggests that oxidative stress that characterizes periodontitis causes an increase in radical scavenging of antioxidant species, and that, removal of oxidative stress restores a normal SOD activity, which is in accordance with study of Takane et al. [24] and Sculley and Langley-Evans [25]. At 2 months post NSPT the median gingival SOD scores of control and test groups were 0.85 and 1.00 respectively, which are accordance with Chapple et al. [13]. The authors reported that local total antioxidant capacity in chronic periodontitis appears to reflect increased oxygen radical activity during periodontal inflammation and can be restored to normal in control subjects by successful periodontal therapy.

However, there are certain limitations in the present study like the smaller sample size. In addition, in this study we have evaluated the total SOD activity level but not the SOD isotypes levels (Mn-SOD, Cu/Zn-
SOD, and EC-SOD). Further long-term studies with large sample sizes are warranted for quantitative assessment of different SOD isotype activity in different severities of chronic periodontitis. In addition, the effect of NSPT on the SOD isotype activity and the identification of SOD isotype variations in chronic and aggressive periodontitis patients needs to be evaluated. Moreover, molecular and cellular studies are required to specify the exact role of SOD in protecting the periodontium.

\section{Conclusion}

There was a significant reduction in clinical parameters (PI, GI, GBI, PD and CAL) after 2 months post NSPT. The SOD activity in gingiva of patients with chronic periodontitis was found to be higher than healthy controls. This increase may be because of increased oxidative stress and increased need for oxidant/anti-oxidant balance for protection. In test group 2 months post NSPT, the gingival SOD levels were close to levels in control group. Also, statistically significant difference was seen in the gingival SOD scores at baseline and at 2 months post therapy, suggesting oxidative stress that characterizes periodontitis causes a reduction in the radical scavenging antioxidant species, and that removal of the oxidative stress by NSPT restores a normal gingival SOD activity. Thus, the effect of NSPT was significant is improving the clinical parameters and restoring the previously increased SOD levels to normal.

\section{Funding}

There is no financial relationship between any author and any commercial firm(s), which may pose a conflict of interest.

\section{References}

[1] Iain LC, Chapple. Reactive oxygen species and antioxidants in inflammatory diseases. J Clin Periodontol 1997; 24:287296.

[2] Akalin FA, Toklu E, Renda N. Analysis of superoxide dismutase activity levels in gingival and gingival crevicular fluid in patients with chronic periodontitis and periodontally healthy controls. J Clin Periodontol 2005; 32:238-243.

[3] Iain LC, Chapple, John B, Matthews. The role of reactive oxygen and antioxidant species in periodontal tissue destruction. Periodontology 2000 2007; 43:160-232. 
[4] Ellis SD, Tucci MA, Serio FG, Johnson RB. Factors for progression of periodontal diseases. J Oral Pathol Med 1998; 27:101-105.

[5] Wei D, Zhang XL, Wang YZ, Yang CX, Chen G. Lipid peroxidation levels, total oxidant status and superoxide dismutase in serum, saliva, Gingival crevicular fluid in chronic periodontitis patients before and after periodontal therapy. Aust Dent $J$ 2010 Mar; 55(1):70-8.

[6] Naofumi T, Takaaki T, Daisuke E, Reiko Y, Tatsuo Y and Manabu M. Short term effects of non surgical periodontal treatment on plasma level of reactive oxygen metabolites in patients with chronic periodontitis. J Periodontol 2009; 80:901-906

[7] Agnihotri R, Pandurang P, Kamath S, Goyal R, Ballal S. Association of cigarette smoking with superoxide dismutase enzyme levels in subjects with chronic periodontitis. J Periodontol 2009; 80:657-662.

[8] Pryor WA. Oxy-radicals and related species. Their formation, lifetimes and reactions. AnnReview of Physiol 1986; 48:657667.

[9] Joanny F, Menvielle-Bourg. Superoxide dismutase (SOD), a powerful antioxidant, is now available orally. Phytothérapie 2005; 3:1-4.

[10] Bennet H, Jacoby, Walter L, Davis. The Electron microscopic immunolocalization of a copper-zinc superoxide dismutase in association with collagen fibres of periodontal soft tissues. $J$ Periodontol 1991; 62:413-420.

[11] Iain LC, Chapple. Role of free radicals and antioxidants in the pathogenesis of the inflammatory periodontal diseases. J Clin Pathol Mol Pathol 1996; 49:M247-M255.

[12] Hei-Jin Na, Ok-Su Kim, Byung-Ju Park. Expression of superoxide dismutase isoforms in inflamed gingival. J Periodontol Implant Sci 2006; 36(1):97-106.

[13] Chapple LC. Reactive oxygen species and antioxidants in inflammatory diseases. J Clin Periodontol 1997; 24:287-296.

[14] Bauer V, Bauer F. Reactive oxygen species as mediator of tissue protedtion and injury. Gen Physiol Biophys 1999; 18:714.
[15] Igaru T, Kaneda H, Horiuchi S and Ono S. A remarkable increase of superoxide dismutase activity in synovial fluid of patients with rheumatoid arthritis. Clinical Orthopaedics Rel Res 1982; 162:282-287.

[16] Kimura F, Hasegawa G, Obayashi H. Serum extracellular superoxide dismutase in patients with type 2 diabetes. Diabetes Care 2003; 26:1246-1250.

[17] Agnihotri R, Pandurang P, Kamath S, Goyal R, Ballal S. Association of cigarette smoking with superoxide dismutase enzyme levels in subjects with chronic periodontitis. J Periodontol 2009; 80:657-662.

[18] Garg N, Singh R, Dixit J, Jain A, Tewari V. Levels of lipid peroxides and antioxidants in smokers and non smokers. $J P e$ riodontal Res 2006; 41:405-410.

[19] Ryder MI, Appel WH, Verhoeven AJ. Alterations of neutrophil oxidative burst by in vitro smoke exposure: Implications for oral and systemic diseases. Ann Periodontol 1998; 3:76-87.

[20] Alfano MC. The origin of gingival fluid. J Theor Biol 1974; 139:43-48

[21] Carranza FA, Newman MG. Clinical Periodontology, WB Saunders \& Co., Philadelphia, $10^{t h}$ Edition, pp. 551-552.

[22] Asman B. Peripheral PMN cells in juvenile periodontitis. $J$ Clin Periodontol 1998; 15:360-364.

[23] Godin DV and Wohaieb SA. Nutritional deficiency, starvation and tissue antioxidant status. Free Radic Biol Med 1988; 5:165-176

[24] Takane M, Sugano N, Iwasaki H. New biomarker evidence of oxidative DNA damage in whole saliva from clinically healthy and periodontally diseased individuals. J Periodontol 2002; 73:551-554

[25] Sculley DV and Langley-Evans SC. Periodontal disease is associated with lower antioxidant capacity in whole saliva and evidence of increased protein oxidation. Clinical Science 2003 ; $105: 167-172$ 


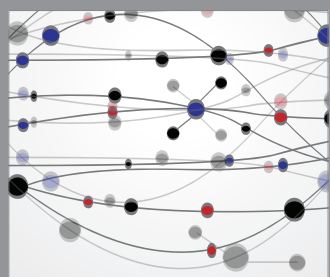

The Scientific World Journal
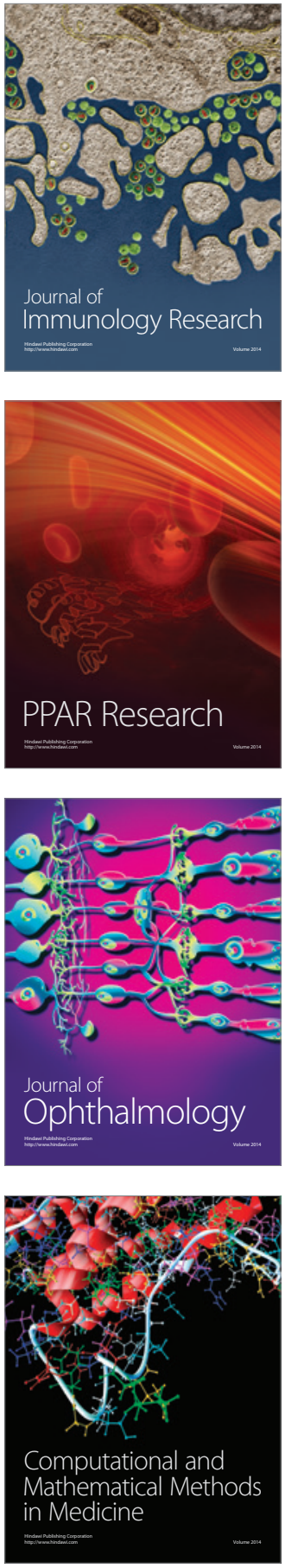

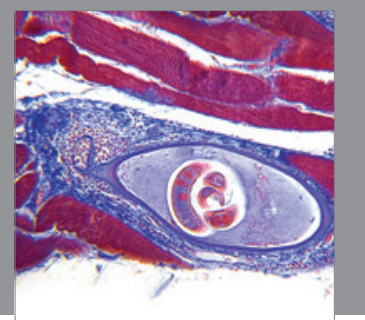

Gastroenterology

Research and Practice
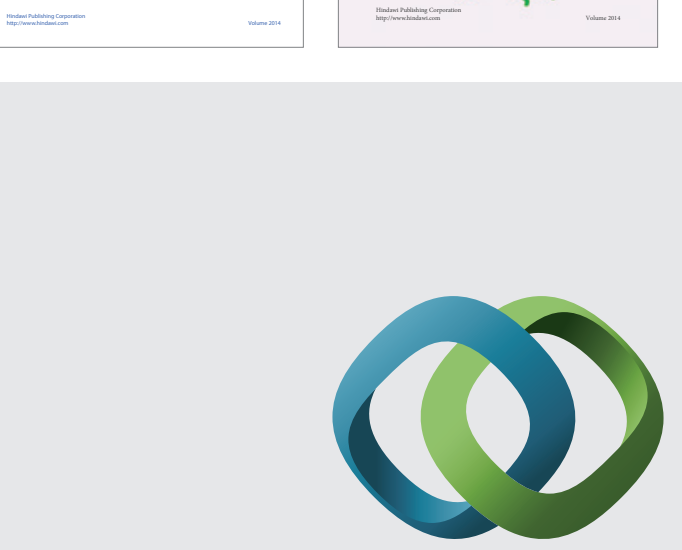

\section{Hindawi}

Submit your manuscripts at

http://www.hindawi.com
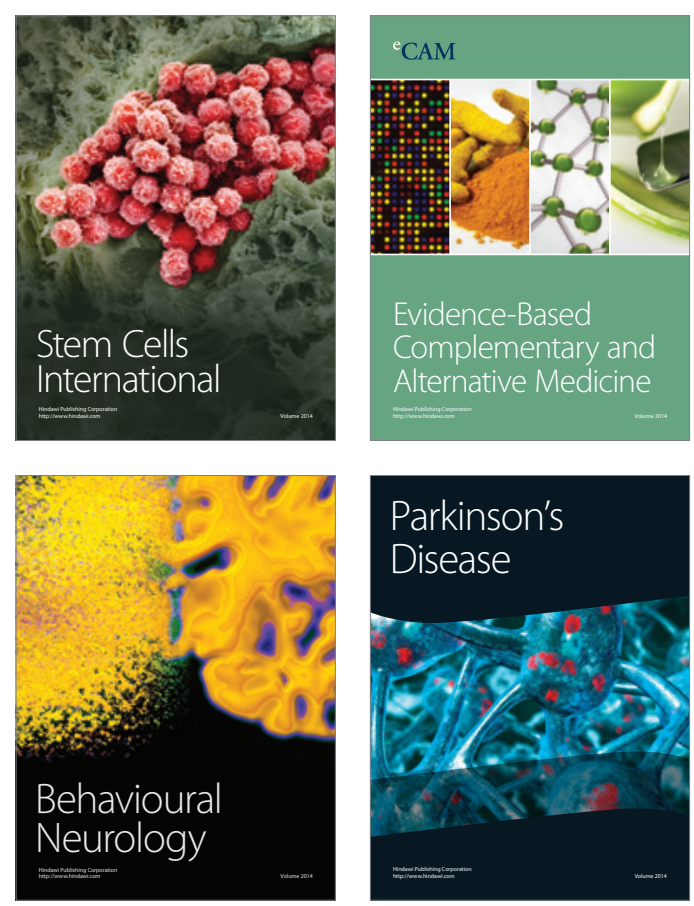

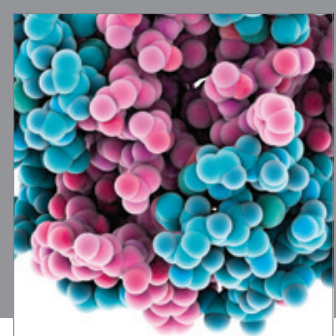

Journal of
Diabetes Research

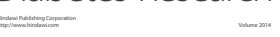

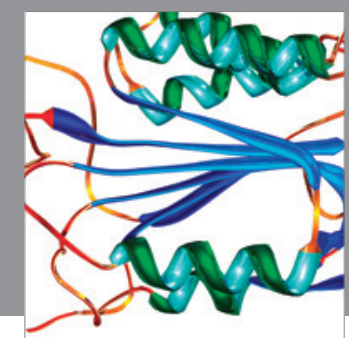

Disease Markers
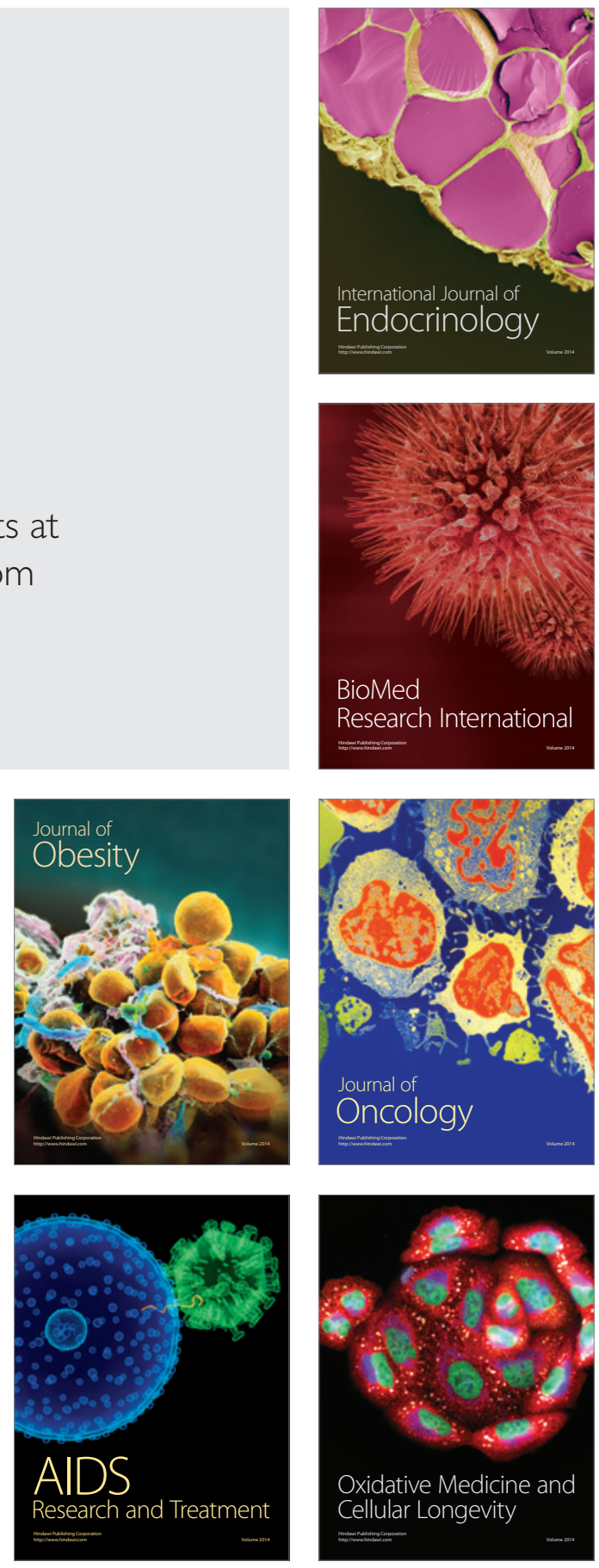